\title{
Double discontinuities at the magnetotail plasma sheet-lobe boundary
}

\author{
Y. C. Whang ${ }^{1}$, D. Fairfield ${ }^{2}$, R. P. Lepping ${ }^{2}$, T. Mukai ${ }^{3}$, Y. Saito ${ }^{3}$, J. Slavin ${ }^{2}$, and A. Szabo ${ }^{2}$ \\ ${ }^{1}$ Catholic University of America, Washington, D.C. 20064, USA \\ ${ }^{2}$ NASA Goddard Space Flight Center, Greenbelt, Maryland, 20771, USA \\ ${ }^{3}$ Institute of Space and Astronautical Science, Yoshinodai, Sagamihara, Kanagawa 229, Japan
}

Received: 11 January 2001 - Revised: 9 July 2001 - Accepted: 23 August 2001

\begin{abstract}
A double discontinuity is a compound structure composed of a slow shock layer and an adjoining rotational discontinuity layer on the postshock side. We use highresolution data from Geotail and Wind spacecraft to examine the interior structure within the finite thickness of the discontinuity at the plasma sheet-lobe boundary and found that recognizable MHD structures at the boundary can be standalone slow shocks or double discontinuities. The plasma density increases significantly and the magnetic field intensity decreases significantly across the interior of the slow shock layer. Through the rotational layer, the magnetic field rotates about the normal direction of the shock surface, as the plasma density and the magnetic field intensity remain nearly unchanged. The rotational angle can vary over a wide range. We notice that the observations of double discontinuities are no less frequent than the observations of stand-alone slow shocks. Identification of slow shocks and double discontinuities infers that plasma and magnetic field lines continuously move across the boundary surface from the lobe into the plasma sheet, and there is a conversion of magnetic field energy into plasma thermal energy through the slow shock layer. The double discontinuities also allows for a rapid rotation of the postshock magnetic field lines immediately behind the shock layer to accommodate the environment of the MHD flow in the plasma sheet region.
\end{abstract}

Key words. Magnetospheric physics (plasma sheet) Space plasma physics (discontinuities; shock waves)

\section{Introduction}

Based on classical theory, there are four kinds of magnetohydrodynamics (MHD) discontinuities: MHD fast or slow shock, rotational discontinuity, tangential discontinuity, and contact discontinuity. There is a mass flow across the MHD shock or rotational discontinuity, but there is no mass flow across the tangential discontinuity or contact discontinuity.

Correspondence to: Y. C. Whang (whang@ cua.edu)
These discontinuities are well understood in theory and their existence in space plasma has been verified using observational data from spacecraft. This research is concerned with the observations of a compound structure composed of a slow shock layer and an adjoining rotational discontinuity layer on the postshock side. Such a compound structure looks like a new kind of MHD discontinuity; it is called a double discontinuity.

The basic identification of an MHD slow shock from observational data is to analyze the observed jump conditions of the plasma and magnetic field on two sides of the shock surface. The data analysis simply treats the shock as a discontinuity. This is, of course, a fundamentally important step. Following this approach, a few dozen slow shocks have been identified in the magnetotail (Feldman et al., 1984, 1985, 1987; Smith et al., 1984; Seon et al., 1995, 1996; and Saito et al., 1995, 1996) and a smaller number of slow shocks have been identified in interplanetary space (Chao and Olbert, 1970; Burlaga and Chao, 1971; Richter et al., 1985; and Whang et al., 1996).

MHD shocks are not discontinuities in the strict sense. A shock has a finite thickness across which physical properties change continuously. This leads to another area of slow shock research that is to study the interior structure within the finite thickness of the shock. The objective is to study the variations in the plasma and magnetic field inside the shock, based on theory or based on observational data. Our recent research along this line based on high-resolution observational data in interplanetary space and in the magnetotail region led to a rather surprising discovery of double discontinuities. We found that a slow shock is often followed by an adjoining rotational discontinuity layer on the postshock side. The thicknesses of the two layers are of the same order of magnitude. The change in magnitude of the magnetic field occurs primarily inside the shock layer. Through the rotational layer, the magnetic field rotates about the normal direction of the shock layer; thus, the magnetic field is no longer coplanar on two sides of a double discontinuity.

Double discontinuities were first identified in interplane- 
tary space from the Wind spacecraft (Whang et al., 1998a; hereafter referred to as Paper 1). In one occasion on 6 April 1995 a double discontinuity observed from the Wind spacecraft was also observed from the Geotail spacecraft in the upstream of the Earth's bow shock. The Geotail spacecraft crossed the double discontinuity $51 \mathrm{~min}$ later than the crossing time of the Wind spacecraft, yet the magnetic field structures observed from the two spacecraft at two locations have nearly identical signatures. During this time interval, the discontinuity surface has traveled over a distance of $>10^{6} \mathrm{~km}$, and the two crossing points on the surface of the observed double discontinuity are at a distance of $>0.4 \times 10^{6} \mathrm{~km}$ apart. These observations imply that double discontinuities in interplanetary space are reasonably stable, large-scale structures. Observation of two double discontinuities in the Earth's magnetotail has also been reported (Whang et al., 1997; hereafter referred to as Paper 2). Since double discontinuities exist not only in interplanetary space but also in the magnetotail region, they could be a general MHD structure in space plasma. This paper is the third journal article dealing with the observations of double discontinuities.

\section{Geotail observations of slow shocks and double dis- continuities}

2.1 Slow shocks identified at the plasma sheet-lobe boundary

During a 5-month period between 14 September 1993 and 16 February 1994, the Geotail spacecraft made 303 crossings of the plasma sheet-lobe boundary in the mid-tail to distant-tail regions of the magnetotail. Saito et al. (1995) studied the observed conditions across the discontinuities at these crossings using the plasma data from the Low Energy Particle (LEP) analyzer (Mukai et al., 1994) and the magnetic field data (Kokubun et al., 1994). They found that the jump conditions at thirty-two boundary crossings satisfy the Rankine-Hugoniot relations of MHD slow shocks.

For each of the 32 slow shocks identified at the plasma sheet-lobe boundary, there is a mass flow entering the shock from the lobe side. The relative flow speeds are $U_{1 n}>C_{s 1}$ on the preshock (lobe) side, $U_{2 n}<C_{s 2}$ on the postshock (plasma sheet) side, and $U_{n}<\alpha \cos \Theta$ on both sides of the shock; here $U_{n}$ is the normal flow speed in the shock frame, $\alpha$ is the Alfvén speed, $C_{s}$ is the slow speed, $\Theta$ is the angle between the shock normal and the magnetic field vector, and the subscripts 1 and 2 denote the conditions on the preshock side and the postshock side.

The time scale for the Geotail spacecraft to traverse the slow shocks identified by Saito et al. (1995) at the plasma sheet-lobe boundary is of the order of 20-60 s. Since these slow shocks are relatively thick MHD discontinuities, it becomes possible to carry out the second phase of the slow shock study to examine the plasma and the magnetic field structure through the interior of these shocks using highresolution data. We have studied the interior structures for some of the 32 slow shocks identified by Saito et al. (1995). We found that some of them are accompanied by an adjoining rotational discontinuity layer on the postshock side and others are stand-alone slow shocks not accompanied by rotational layers; the discontinuity at the plasma sheet-lobe boundary can be a slow shock or a double discontinuity. Next, we will show examples of one stand-alone slow shock, one double discontinuity with a large rotational angle, and one double discontinuity with a small rotational angle.

\subsection{A double discontinuity observed on 14 February 1994}

On 14 February 1994, the Geotail spacecraft crossed the boundary surface entering the plasma sheet from the north lobe of the magnetotail at $t=17: 15, X_{G S M}=$ $-54 R_{E}, Y_{G S M}=1.4 R_{E}$ and $Z_{G S M}=-3 R_{E}$. The spacecraft took $58 \mathrm{~s}$ to traverse the thickness of the double discontinuity. The data rate of the magnetic field is 16 data per second: the data have outstanding time-resolutions to examine the detailed magnetic field structure through the interior of a double discontinuity.

From the top panel, we see that a significant decrease in the magnitude of the magnetic field occurs inside a slow shock layer (S-layer) between 17:14:55 and 17:15:41. The next two panels show the longitude and latitude angles

$$
\phi=\arctan \left(B_{y} / B_{x}\right) \quad \theta=\arctan \left(B_{z} / \sqrt{B_{x}^{2}+B_{y}^{2}} .\right.
$$

Here, $B_{x}, B_{y}, B_{z}$ are the three components of the magnetic field in geocentric solar ecliptic (GSE) coordinates. The S-layer is followed by an adjoining rotational discontinuity layer (R-layer) on the postshock side between 17:15:41 and 17:15:53, across which the direction of the magnetic field makes a significant change, while its magnitude almost remains constant. Let $\mathbf{n}=n_{x} \mathbf{i}+n_{y} \mathbf{j}+n_{z} \mathbf{k}$ be the unit normal vector of the shock layer pointing in the direction of the mass flow. The divergence free condition of magnetic field requires that along the $n$-direction, the variation in the magnetic field component should be a minimum. An analysis of the minimal variation for the magnetic field data across the shock layer estimates that the three directional angles of the unit normal vector are $\cos ^{-1} n_{x}=120^{\circ}, \cos ^{-1} n_{y}=139^{\circ}$, and $\cos ^{-1} n_{z}=115^{\circ}$. Through the shock layer, $B_{n}$ remains nearly constant, and the standard deviation of $B_{n}$ is $0.17 \mathrm{nT}$. The mathematical method of determining the shock normal is described in the Appendix.

We use a $n, t, s$ coordinate system to study the variations in the magnetic field through the compound structure. $B_{t}$ and $B_{s}$ are the two components of the magnetic field parallel to the surface of the slow shock layer. The variations in $B_{n}$, $B_{t}$ and $B_{s}$ across the compound structure are plotted in the three lower panels of Fig. 1. The variations in $B_{t}$ and $B_{s}$ inside the R-layer are represented by filled squares in Fig. 2; they show that through the R-layer, the magnetic field rotates by an angle of $\sim 58^{\circ}$ about the normal direction of the shock surface while its magnitude remains nearly constant. The arrow in the figure indicates the direction of field rotation. 


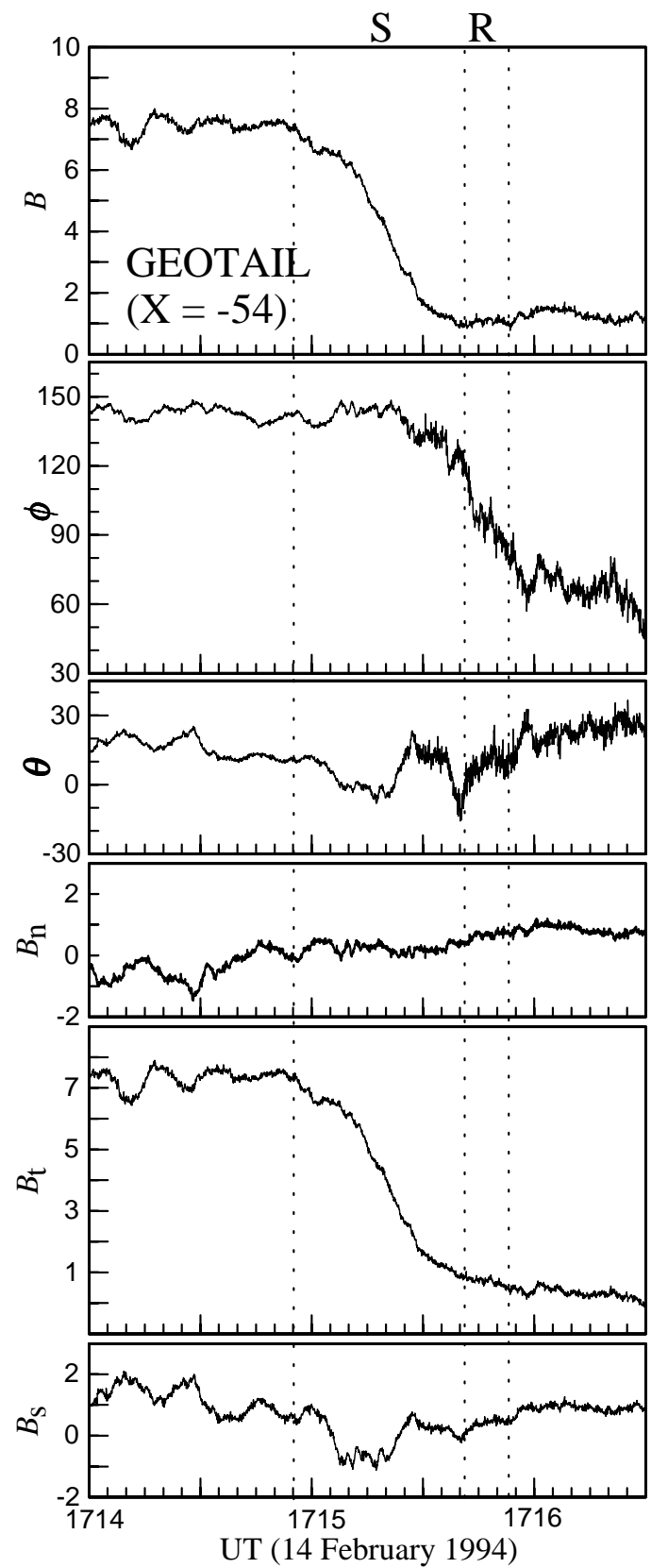

Fig. 1. The observed double discontinuity of 14 February 1994 composed of a slow shock (S-layer) and an adjoining rotational discontinuity (R-layer). The decrease in the magnetic field intensity occurs primarily inside the S-layer. Through the R-layer, the direction of the magnetic field makes a significant change, while its magnitude remains almost constant.

The start and end times of the S-layer and the R-layer are determined by using an iteration procedure. For a given set of start and end times, we first calculate the normal direction of the shock using high-resolution magnetic field data inside the shock layer based on the minimum variance analysis; then we worked simultaneously on all variables using various diagrams shown in Figs. 1 and 2. We adjust the start and end times of the discontinuity layers until a reasonable

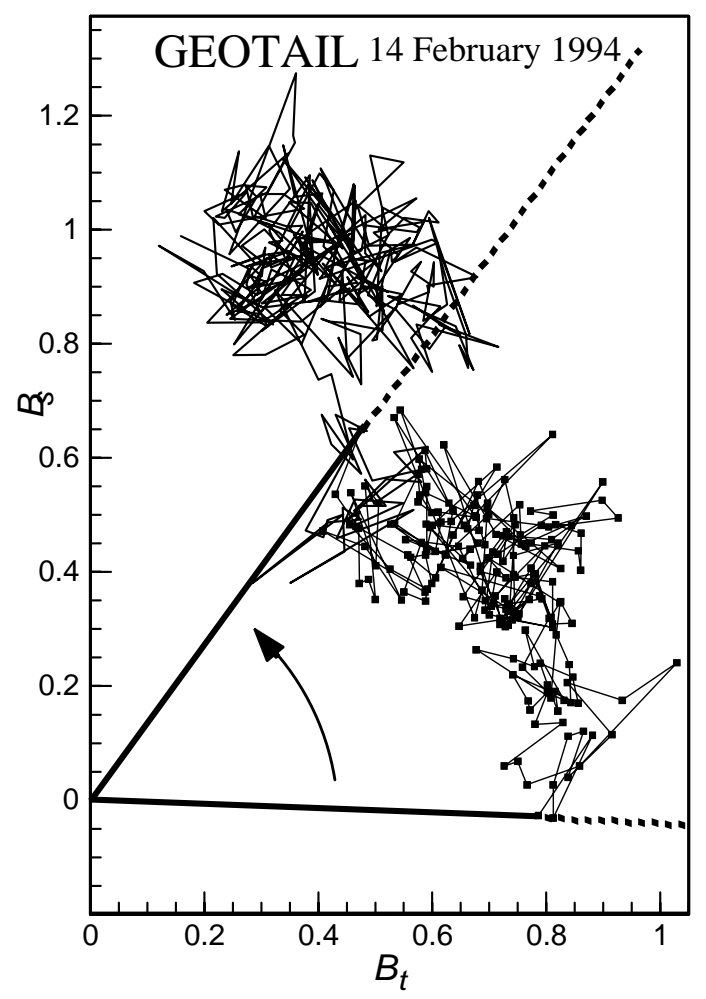

Fig. 2. Filled squares are data points across the R-layer. They show that the magnetic field rotates by an angle of $\sim 58^{\circ}$ about the normal direction of the shock surface, while its magnitude remains nearly constant. The arrow indicates the direction of field rotation. Thin lines are data points during an extended period of 20 $\mathrm{s}$ in the post R-layer region.

picture of the compound structure emerges. The timing at each boundary determined by this procedure is not necessarily a single, clearly defined time. Sometimes the determination for the end time of the R-layer is a little ambiguous. As an example, shown in Fig. 2, we extend the end time of the R-layer by $20 \mathrm{~s}$ and use thin lines to show the variations in $B_{t}$ and $B_{s}$ over the extended period. Inspection of the extended plot may suggest that the rotation angle of the R-layer could be revised by several degrees in either direction. However, under these circumstances, the transition region still fits well with the qualitative description of a compound structure, composed of a slow shock layer and an adjoining rotational discontinuity layer on the postshock side. The magnetic field data for the double discontinuity of 14 February 1994 show that the time scales of the slow shock layer (S-layer) and the adjoining rotational discontinuity layer (R-layer) are $46 \mathrm{~s}$ and $12 \mathrm{~s}$, respectively. Figure 3 shows $150 \mathrm{~s}$ of plasma number density from the Geotail LEP experiment. The top panel shows the electron number density $N_{e}$. These are uncalibrated 3 s electron data calculated onboard every spin period; the data have sufficient time-resolution to describe the variation in $N_{e}$ through the interior of the double discontinuity. $N_{e}$ increases monotonically inside the S-layer, and remains nearly unchanged through the R-layer. The $12 \mathrm{~s}$ average ion 


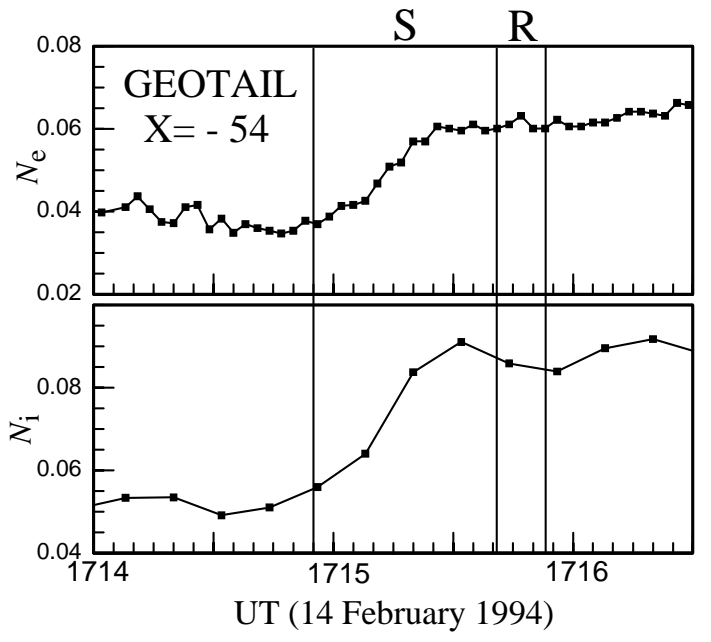

Fig. 3. The top panel shows the $3 \mathrm{~s}$ electron number density $N_{e}$ and the lower panel shows the $12 \mathrm{~s}$ average ion number density data. The plasma density increases monotonically across the slow shock layer.

number density in the lower panel describes the increase in $N_{i}$ inside the S-layer. Across the shock layer, the plasma number density increases by a factor of $\sim 1.7$. The observed plasma and magnetic field structures of the compound structure are consistent with the interpretation that the S-layer is a slow shock layer and the R-layer is a rotational discontinuity layer.

\subsection{A stand-alone slow shock observed on 12 January 1994}

Our study of the interior structure of slow shocks identified by Saito et al. (1995) from Geotail data discovers that the observed discontinuity can be a stand-alone slow shock or a double discontinuity. This means a slow shock at the plasma sheet-lobe boundary is not always followed by a rotational discontinuity. This example presents the interior structure of a stand-alone slow shock. On 12 January 1994, the Geotail spacecraft crossed the slow shock, entering the south lobe from the plasma sheet at $t=15: 49, X_{G S M}=-93 R_{E}$, $Y_{G S M}=14 R_{E}$, and $Z_{G S M}=-3 R_{E}$. The spacecraft took $41 \mathrm{~s}$ to traverse the thickness of the slow shock. We study the magnetic field structure of the slow shock by using highresolution data from Geotail. The three directional angles of the unit normal vector are $\cos ^{-1} n_{x}=94^{\circ}, \cos ^{-1} n_{y}=45^{\circ}$, and $\cos ^{-1} n_{z}=45^{\circ} . B_{n}$ remains nearly constant across the interior of the shock; the standard deviation of $B_{n}$ inside the shock layer is $0.59 \mathrm{nT}$. For a stand-alone slow shock, the magnetic field vectors are essentially parallel to a coplanarity plane; we also calculated the normal to the coplanarity plane. We determine the $s$-coordinate by requiring that the average $B_{s}$ inside the shock layer be zero. The direction of the $t$-coordinate is determined by requiring that $B_{t}$ be positive and it decreases rapidly inside the shock layer. As shown in Fig. 4, both the magnitude and the tangential component of the magnetic field, $B$ and $B_{t}$, decrease monotoni-

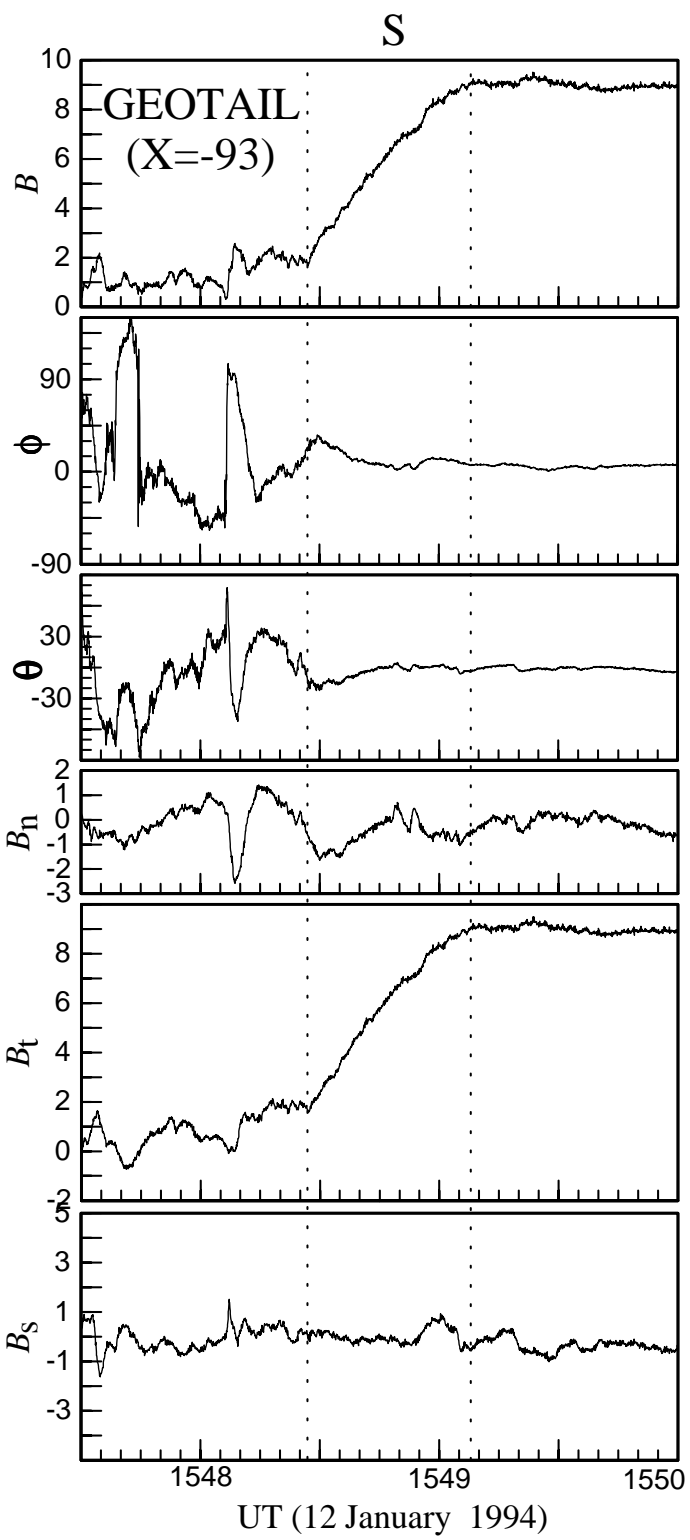

Fig. 4. A stand-alone slow shock was observed on 12 January 1994. The magnetic field vectors inside the shock layer are essentially parallel to a coplanarity plane. Both the magnitude and the tangential component of the magnetic field, $B$ and $B_{t}$, decrease monotonically through the shock layer.

cally through the shock layer. However, the important feature of double discontinuities is the rotation of the magnetic field about the $n$-direction through the R-layer; the magnetic field vectors are not coplanar on two sides of a double discontinuity. The exact directions of the $s, t$ coordinates are less important for a typical double discontinuity. We do not require that the average $B_{s}$ be zero inside the shock layer. Figure 5 shows $150 \mathrm{~s}$ of plasma density data through the slow shock. The top panel shows the $3 \mathrm{~s}$ electron number density $N_{e}$ and the lower panel shows the $12 \mathrm{~s}$ average ion number density $N_{i}$. Across the shock layer, the plasma number density increases monotonically, with the number density increasing 


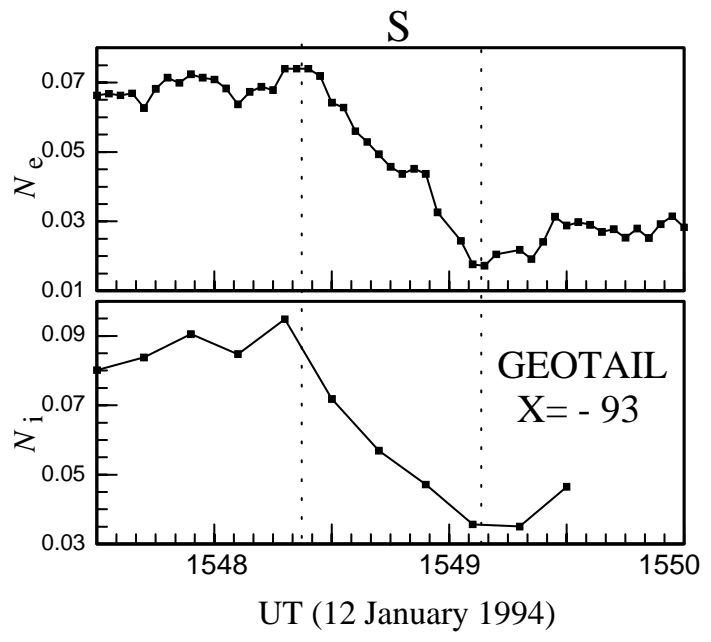

Fig. 5. The structure of plasma density observed inside a standalone slow shock.

by a factor of $\sim 2.8$ (see Whang et al., 1998b, for a similar study of a slow shock in interplanetary space).

\subsection{A slow shock accompanied by a weak rotational layer observed on 23 January 1994}

On 23 January 1994, the Geotail spacecraft crossed the boundary surface to enter the south lobe from the plasma sheet at $t=20: 45, X_{G S M}=-44 R_{E}, Y_{G S M}=$ $-16 R_{E}$, and $Z_{G S M}=-10 R_{E}$. A double discontinuity with small rotation angle was observed at the boundary crossing. Figure 6 shows $150 \mathrm{~s}$ of high-resolution magnetic field data. The time scale for the Geotail spacecraft to traverse the double discontinuity layer is $\sim 25 \mathrm{~s}$. A significant decrease in the magnitude of the magnetic field occurs inside the slow shock layer (S-layer) between 20:45:31 and 20:45:51. The three directional angles of the unit normal vector are $\cos ^{-1} n_{x}=78^{\circ}, \cos ^{-1} n_{y}=159^{\circ}$, and $\cos ^{-1} n_{z}=73^{\circ}$; the standard deviation of $B_{n}$ through the $\mathrm{S}$-layer is $0.26 \mathrm{nT}$. The S-layer is accompanied by an adjoining rotational discontinuity layer (R-layer) on the postshock side between 20:45:26 and 20:45:31, across which the direction of the magnetic field makes a significant change, while its magnitude remains nearly constant.

The variations in $B_{t}$ and $B_{s}$ across the R-layer plotted in Fig. 7 show that through the rotational layer, the magnetic field rotates by a small angle of $\sim 13^{\circ}$ about the normal direction of the shock surface, while its magnitude remains nearly constant.

This discontinuity is one of the 32 slow shock crossings identified by Saito et al. (1995). There is a mass flow entering the shock from the lobe side, and the flow conditions on two sides of the shock satisfy the Rankine-Hugoniot relations. Figure 8 shows $75 \mathrm{~s}$ of plasma number density. $N_{e}$ increases monotonically inside the S-layer, and remains nearly unchanged through the R-layer. The increase in the $12 \mathrm{~s}$ ion number density $N_{i}$ inside the S-layer is plotted in the lower
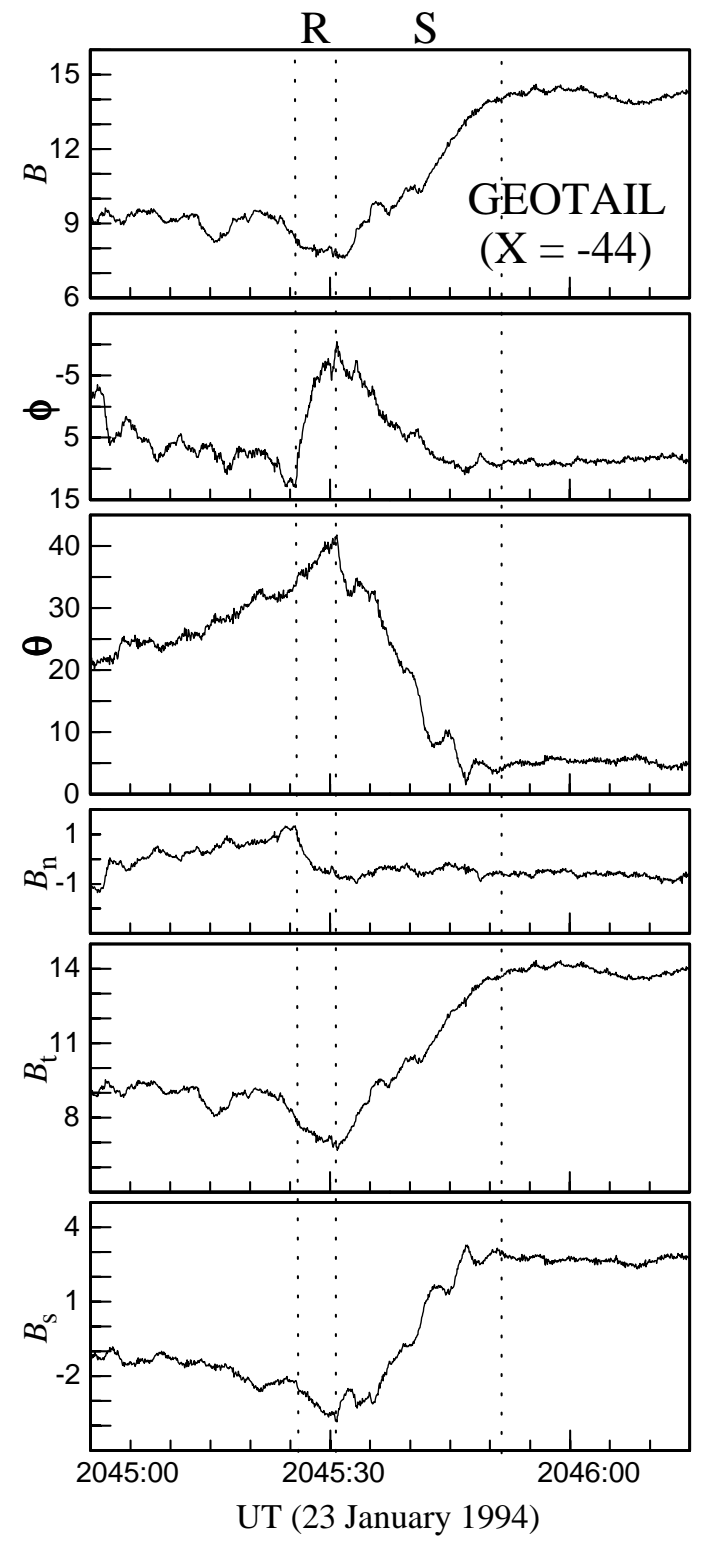

Fig. 6. A double discontinuity was observed on 12 January 1994 from Geotail spacecraft at the plasma sheet-lobe boundary. The Slayer is accompanied by an adjoining rotational discontinuity layer (R-layer) on the postshock side.

panel. Across the shock layer, the plasma number density increases by a factor of $\sim 2.5$. The slow shocks at the plasma sheet-lobe boundary can be a stand-alone slow shock or a double discontinuity that is a slow shock accompanied by a rotational discontinuity (the rotational angle can vary over a wide range). A stand-alone slow shock amounts to a double discontinuity in the limit of a very small rotational angle; thus, slow shocks can actually be considered as a subset of double discontinuities. 


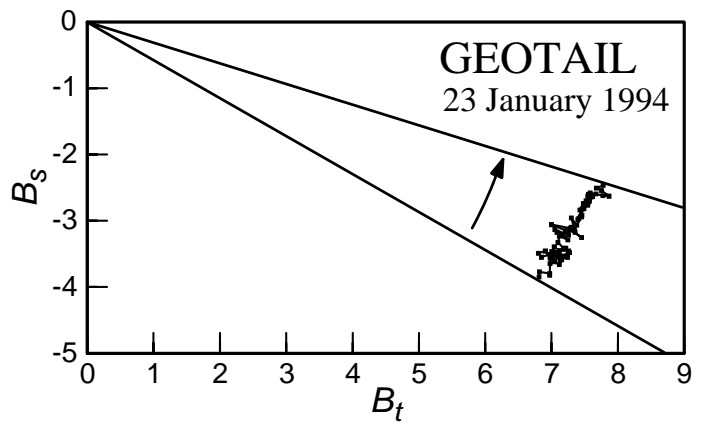

Fig. 7. Through the rotational layer of the double discontinuity shown in Fig. 6, the magnetic field rotates by an angle of $\sim 13^{\circ}$ about the normal direction of the shock surface, while its magnitude remains nearly constant.

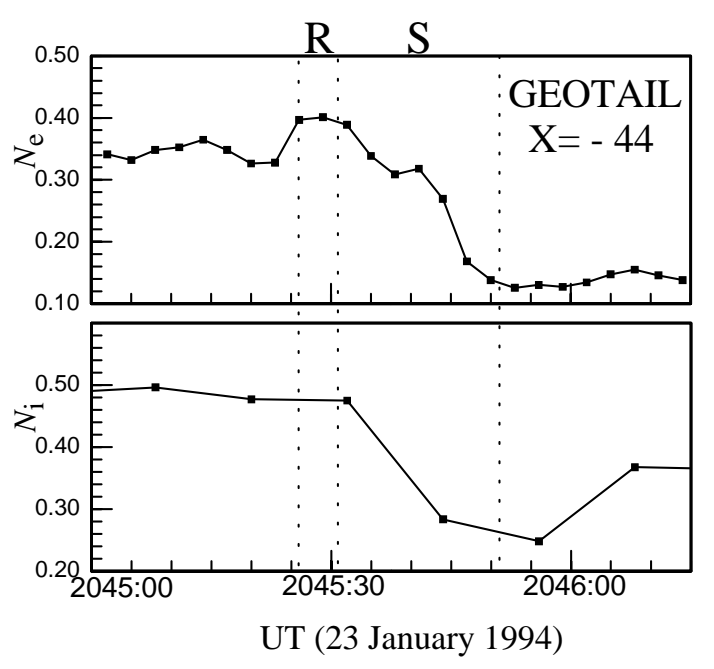

Fig. 8. The plots of the $3 \mathrm{~s}$ electron number density $N_{e}$ and the $12 \mathrm{~s}$ average ion number density $N_{i}$ show that the plasma density increases monotonically inside the S-layer, and remains nearly unchanged through the R-layer.

\section{Wind observations of double discontinuities on 2-5 January 2000}

Between 2 and 5 January 2000, over an 80-hour period, the petal trajectory of the Wind spacecraft in the magnetotail was very close to the boundary between the plasma sheet and the south lobe (Fig. 9). Due to the tail flapping motion, more than one dozen slow shocks and double discontinuities were identified at the plasma sheet-lobe boundary from the Wind magnetic field instrument. In this section, we present four examples of double discontinuities, one on each day, using 92 milliseconds magnetic field data (Lepping et al., 1995). These double discontinuities were observed at $X_{G S E}=-44 R_{E},-48 R_{E},-62 R_{E}$, and $-66 R_{E}$, respectively. The double discontinuity of 4 January 2000 was observed when the spacecraft crossed the boundary surface entering the south lobe from the plasma sheet; the double discontinuities of 2, 3 and 5 January were observed when the

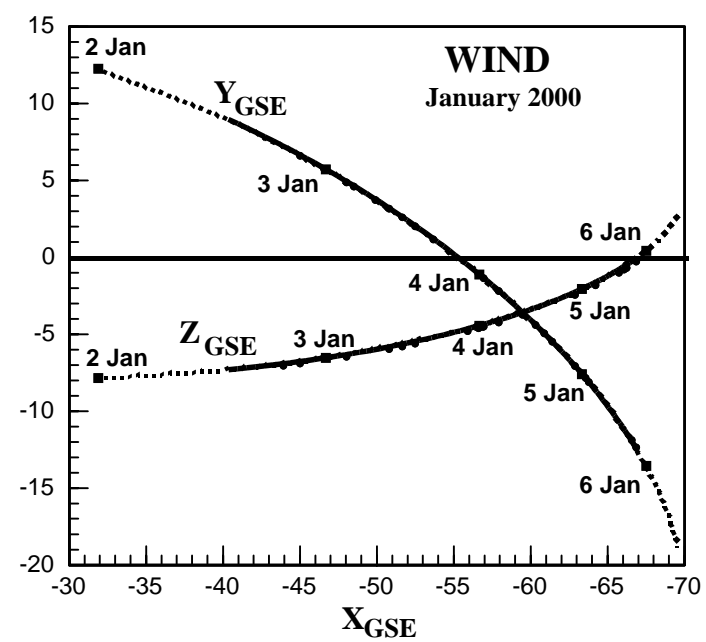

Fig. 9. The solid curves show the petal trajectory of the Wind spacecraft in the magnetotail during an 80-hour period on 2-5 January 2000. The trajectory was very close to the boundary between the plasma sheet and the south lobe.

spacecraft crossed the boundary surface entering the plasma sheet from the south lobe.

The analyses of magnetic field data of the four double discontinuities are shown in Figs. 10 to 13. The three top panels in each figure show the field magnitude, the longitude and latitude angles. The spacecraft observed large changes in the magnetic field intensity through the slow shock layers (S-layer), and large changes in the longitude and latitude angles through the adjoining rotational discontinuity layers (Rlayer). The variations in $B_{n}, B_{t}$, and $B_{s}$ are plotted in the three lower panels of each figure. $B_{n}$ remains nearly constant across the slow shock layer. The standard deviations in $B_{n}$ of the four cases are $0.05 \mathrm{nT}, 0.24 \mathrm{nT}, 0.07 \mathrm{nT}$, and $0.13 \mathrm{nT}$, respectively.

The variations in $B_{t}$ and $B_{s}$ across the R-layer of each compound structure are plotted in Fig. 14 to show that the magnetic field rotates about the normal direction of the shock surface through the R-layer, while its magnitude remains nearly constant. The rotation angles of the four double discontinuities are $18^{\circ}, 27^{\circ}, 95^{\circ}$, and $98^{\circ}$, respectively. The arrow in each plot indicates the direction of field rotation.

During the period of observation, the time resolution of plasma data available from Wind's 3-D Plasma and Energetic Particles Instrument (3DP) are not high enough to show the interior structure of these double discontinuities. Another difficulty associated with the 3DP data is the domination of instrumental effects over the very low particle flux in the lobe. Thus, we are not able to investigate the plasma conditions for these double discontinuities. However, since the rotation of the magnetic field through the R-layer is accompanied by a mass flow across the compound structure and although we cannot investigate the Rankine-Hugoniot conditions, there is no possibility that the observed discontinuities could be tangential or contact discontinuities. 

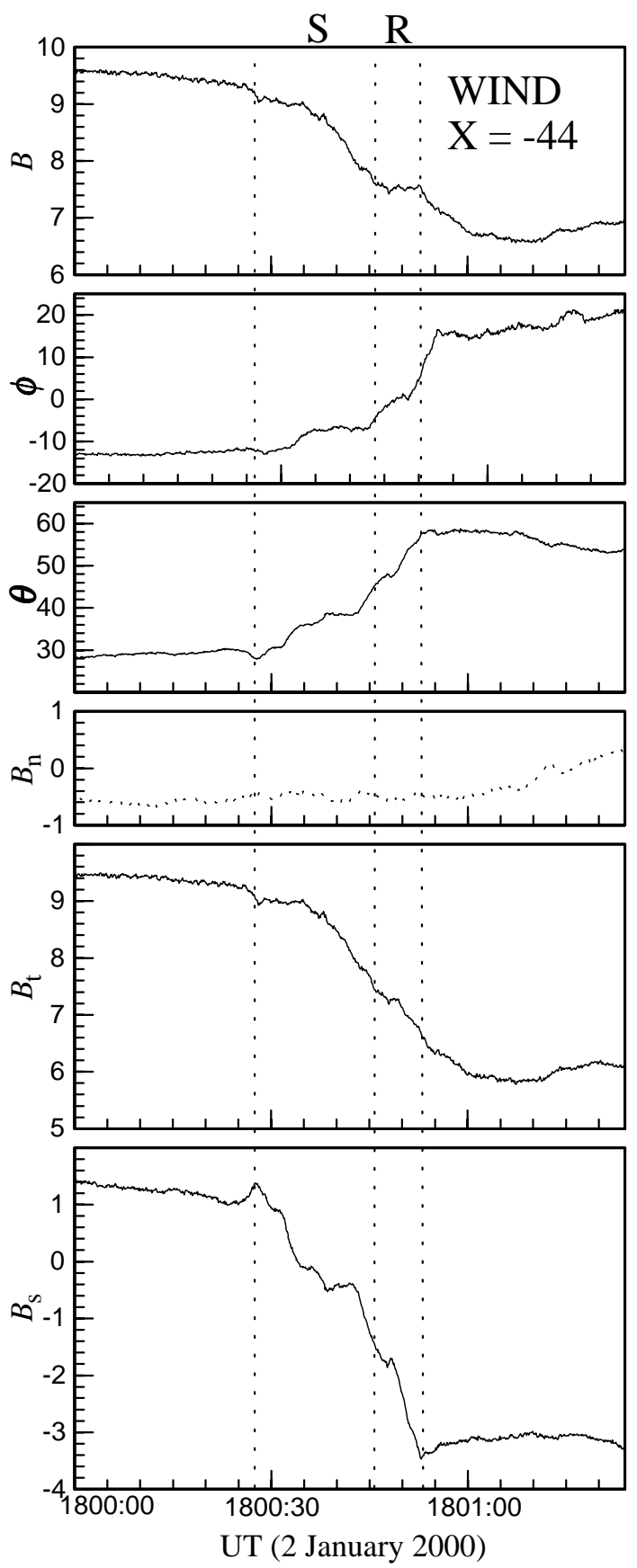

Fig. 10. A double discontinuity was observed on 2 January 2000 when the Wind spacecraft crossed the boundary surface entering the plasma sheet from the south lobe. This figure shows the magnetic field structure of the double discontinuity observed from highresolution Wind data.

\section{Summary and discussions}

When high-resolution data are used to examine the detailed structure inside a slow shock at the plasma sheet-lobe boundary, we find that the slow shock layer is often accompanied by an adjoining rotational discontinuity layer on the postshock (plasma sheet) side. Such a compound structure

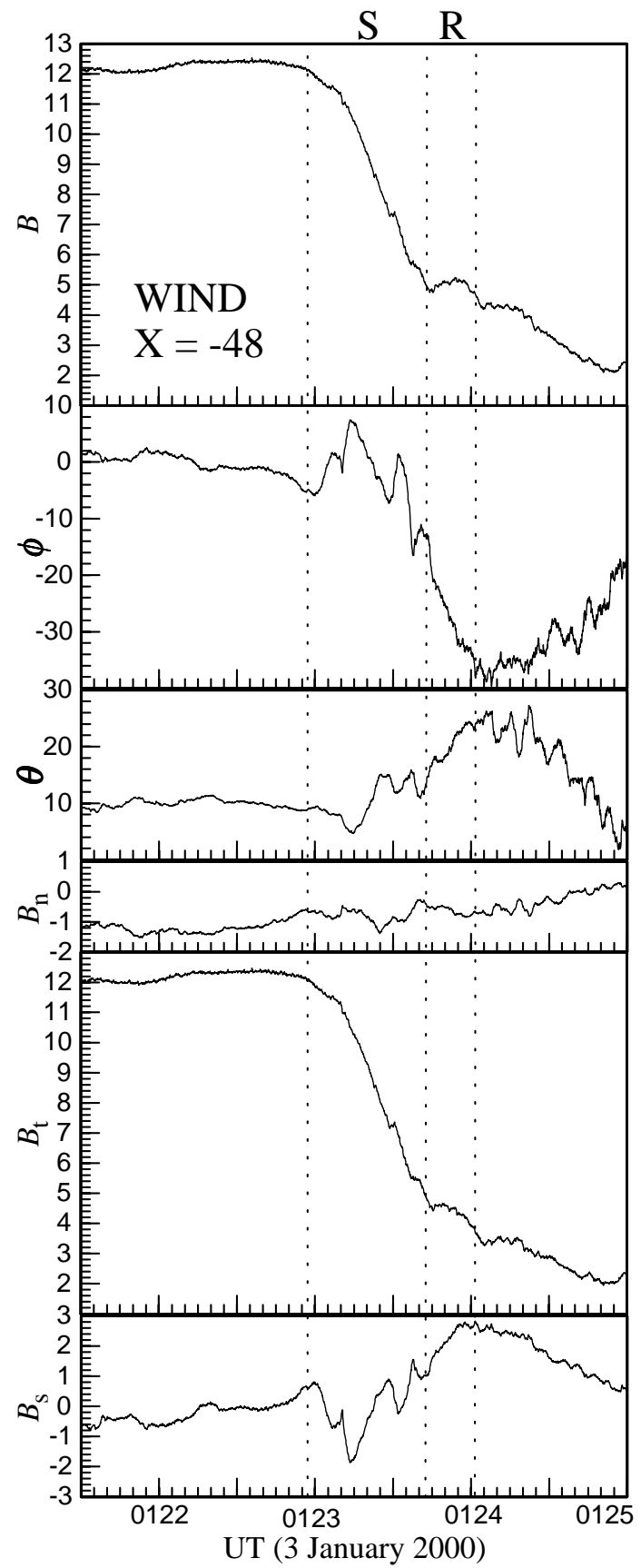

Fig. 11. A double discontinuity observed from Wind on 3 January 2000. The observed structure is composed of a slow shock layer (Slayer) and an adjoining rotational layer (R-layer) on the postshock side.

is known as a double discontinuity. The plasma density increases significantly and the magnetic field intensity decreases significantly across the interior of the slow shock layer. Through the rotational layer, the magnetic field rotates about the normal direction of the shock surface, as the plasma density and the magnetic field intensity remain nearly unchanged. The rotation can be clockwise or counterclockwise. Table 1 presents a summary of double discontinuities 


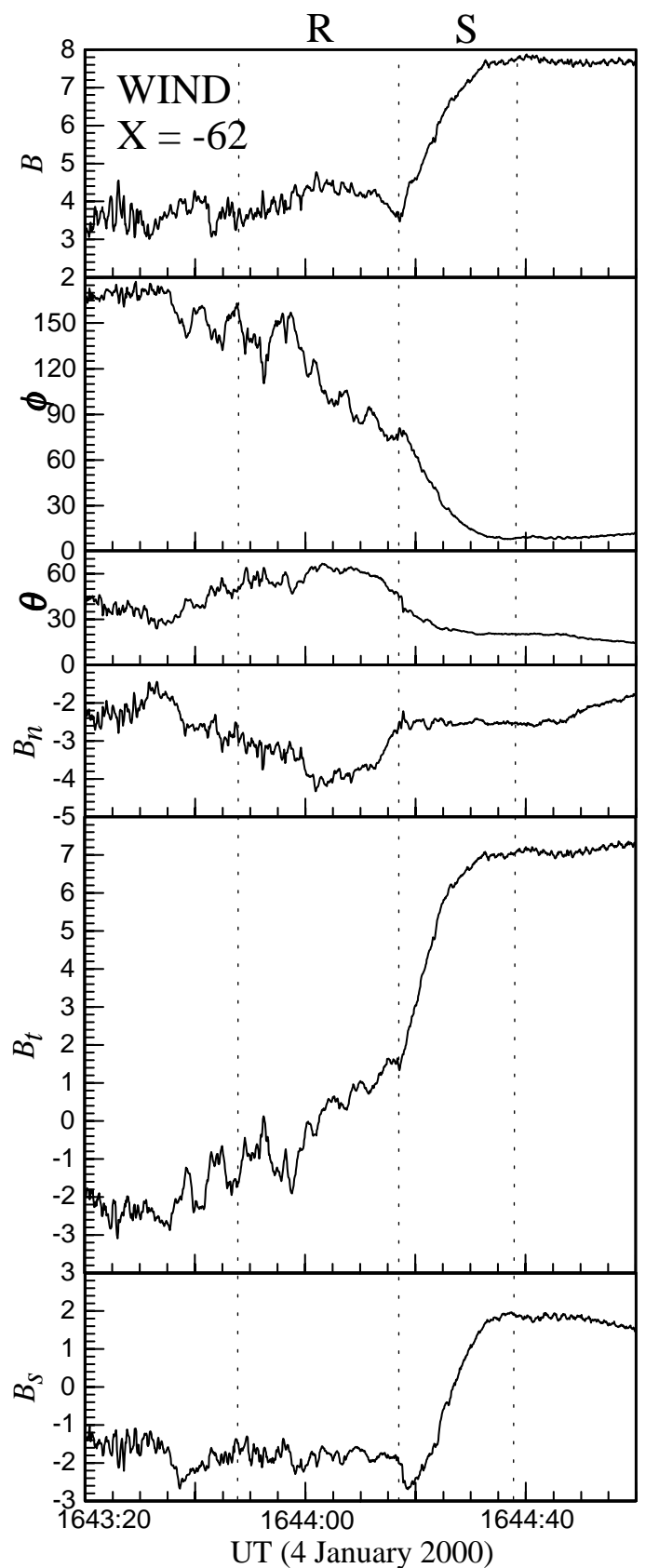

Fig. 12. The double discontinuity of 4 January 2000 was observed when the spacecraft crossed the boundary surface entering the south lobe from the plasma sheet.

in the magnetotail that have been documented in journal articles. All observed double discontinuities have large shock angles $\Theta_{1}$ on the preshock (lobe) side.

The first method to identify slow shocks from observational data is to analyze the observed jump conditions of the plasma and magnetic field on two sides of the shock discontinuity. Another approach for studying slow shocks is to analyze the interior structure within the finite thickness of a slow shock using high-resolution data. Only the second method can identify a double discontinuity. Recognizable
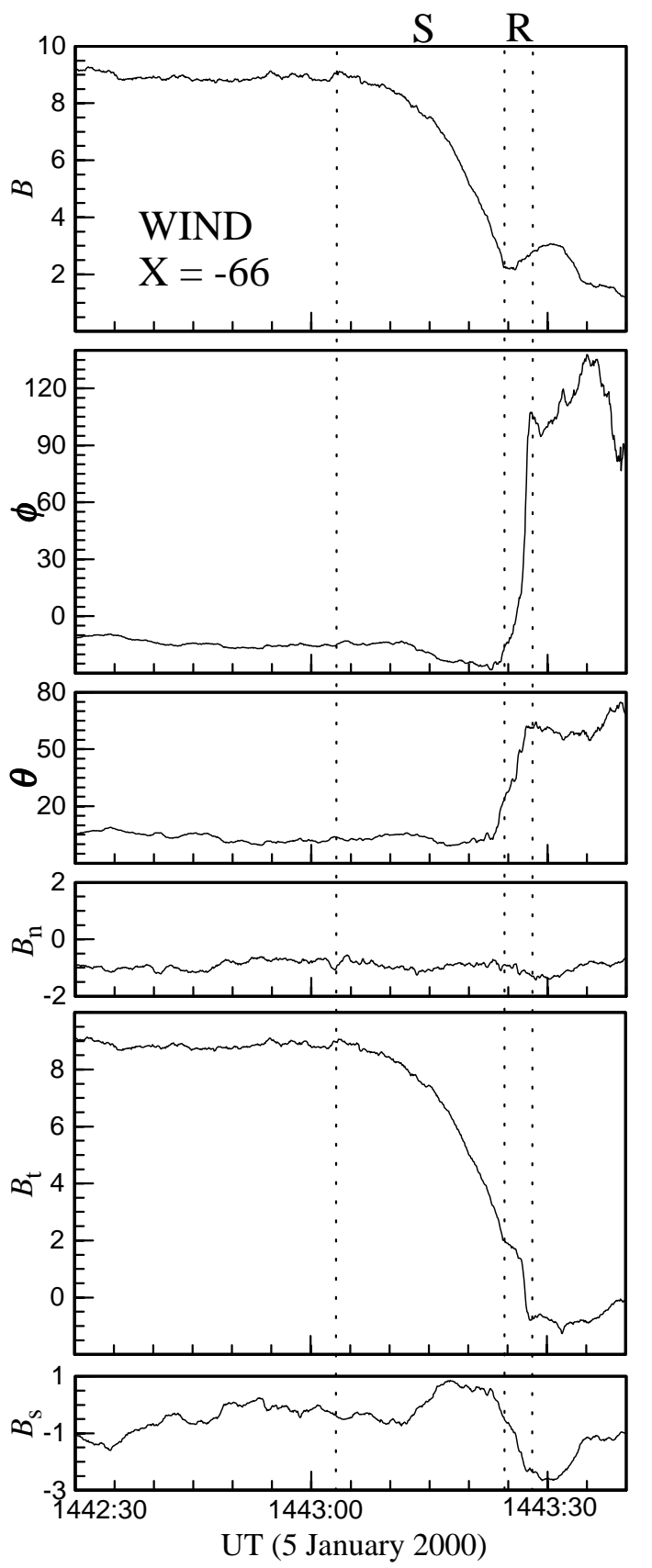

Fig. 13. More than a dozen slow shocks and double discontinuities were identified at the plasma sheet-lobe boundary during an 80-hour period on 2-5 January 2000. This figure shows the magnetic field structure of the 5 January double discontinuity.

MHD structures at the plasma sheet-lobe boundary can be stand-alone slow shocks or double discontinuities. The angle through which the magnetic field rotates across the rotational layer can vary over a wide range. Within the limit of a very small rotational angle, a double discontinuity reduces to a stand-alone slow shock. Thus, slow shocks can actually be considered as a subset of double discontinuities. We notice that in interplanetary space and in the magnetotail, the observations of double discontinuities are no less frequent than the 
Table 1. A summary of double discontinuities in the magnetotail that have been documented in journal articles

\begin{tabular}{cccccc}
\hline $\begin{array}{c}\text { Location } \\
X_{G S E}\left(R_{E}\right)\end{array}$ & $\begin{array}{c}\text { Date of } \\
\text { observation }\end{array}$ & $\begin{array}{c}\text { Rotation } \\
\text { angle degree }\end{array}$ & $\begin{array}{c}\text { Shock } \\
\text { angle } \Theta_{1} \text { degrees }\end{array}$ & Spacecraft & $\begin{array}{c}\text { Documentation of } \\
\text { data analysis }\end{array}$ \\
\hline-44 & 23 January 1994 & 13 & 88 & Geotail & This paper \\
-44 & 2 January 2000 & 18 & 87 & Wind & This paper \\
-48 & 3 January 2000 & 27 & 84 & Wind & This paper \\
-54 & 14 February 1994 & 58 & 89 & Geotail & This paper \\
-62 & 4 January 2000 & 95 & 83 & Wind & This paper \\
-66 & 5 January 2000 & 98 & 84 & Wind & This paper \\
-92 & 17 January 1994 & 67 & 85 & Geotail & Paper 2 \\
-220 & 2 February 1983 & 135 & 88 & ISEE 3 & Paper 2 \\
\hline
\end{tabular}

observations of stand-alone slow shocks.

During a 25-day interval, between 18 January and 11 February 1983, twenty-six slow shocks were identified from ISEE 3 data in the distant tail. Based on these observations, Feldman et al. (1985) concluded that slow shocks are a quasi-permanent structure at the plasma sheet-lobe boundary. On the other hand, during a 5-month interval between, 14 September 1993 and 16 February 1994, Geotail observations in the mid-tail to distant-tail revealed that only about $10 \%$ of the boundary crossings could be identified as slow shocks and double discontinuities. In Sect. 3, we report that over an 80-hour interval, between 2 and 5 January 2000, more than one dozen slow shocks and double discontinuities were identified at the boundary along the Wind petal trajectory in the mid-tail. It appears that the Wind observations incline to support the conclusion of the ISEE 3 observation. Slow shocks and double discontinuities could be a semi-permanent structure at the plasma sheet-lobe boundary.

Early works on the lobe-plasma sheet transition include the formation of a pair of slow shocks (Petschek, 1964; Vasyliunas, 1975) and the formation of a pair of rotational discontinuities (Sonnerup, 1970) (see Priest, 1982 for a review of these relevant works). Observations of slow shocks and double discontinuities reported in this paper infer that plasma and magnetic field lines continuously move across the boundary surface from the lobe region into the plasma sheet region, and there is a conversion of magnetic field energy into plasma thermal energy through the slow shock layer. The double discontinuities at the boundary also allows for a rapid rotation of the postshock magnetic field lines immediately behind the shock layer to accommodate the environment of the MHD flow in the plasma sheet region.

Seon et al. (1996) have described variations in magnetic fields that could be considered as circularly polarized structures in the shock layer in some slow shock crossings in the distant magnetotail. Song et al. (1992) have reported the observation of a possible superposition of a rotational discontinuity and slow mode discontinuity in front of the magnetopause. These two research examples may have been observations of double discontinuities. The component of the magnetic field that deviates from the plane, defined by the shock normal and the preshock magnetic field for fast shocks, has been examined by a number of authors (Thomsen et al., 1987; Jones and Ellison, 1987; Gosling et al., 1988; Friedman et al., 1990). It is extremely interesting if they can use high-resolution observational data to show that a rotational discontinuity layer may also exist immediately adjacent to a fast shock.

Based on the coplanarity theory, Colburn and Sonett (1966) and Abraham-Shrauner and Yun (1976) have studied the method to calculate the shock normal using the average vector properties ( $\boldsymbol{B}$ or $\boldsymbol{B}$ and $\boldsymbol{V}$ ) on two sides of an MHD shock. The input vectors are obtained from observed solar wind data averaged over a given set of time intervals on each side of the shock. Once there is a noncoplanar component of the magnetic field or a rotational discontinuity layer immediately adjacent to a shock, we propose that if high-resolution magnetic field data are available inside the shock layer then one could use the minimum variance analysis to calculate the shock normal.

Observational evidence for the existence of double discontinuities in space plasmas seems to progress far ahead of the theoretical understanding. According to the classical MHD theory, the rotational layer should be penetrating through the slow shock layer from behind and then continue to propagate away from the slow shock layer in the preshock region as a separate MHD discontinuity. However, observed double discontinuities in interplanetary space and in the magnetotail clearly show that under certain conditions, the slow shock layer and the rotational discontinuity layer merge to form a stable compound structure (Paper 1); there is no penetration. Presently, not enough observational data are available to determine the conditions under which double discontinuities could exist. Recently, Lee et al. (2000) carried out a series of hybrid simulations to examine the interaction of a rotational discontinuity with a slow shock in anisotropic plasma. The simulation results cannot reproduce the formation of a double discontinuity in which a rotational layer appears in the immediate postshock side of a slow shock layer. 

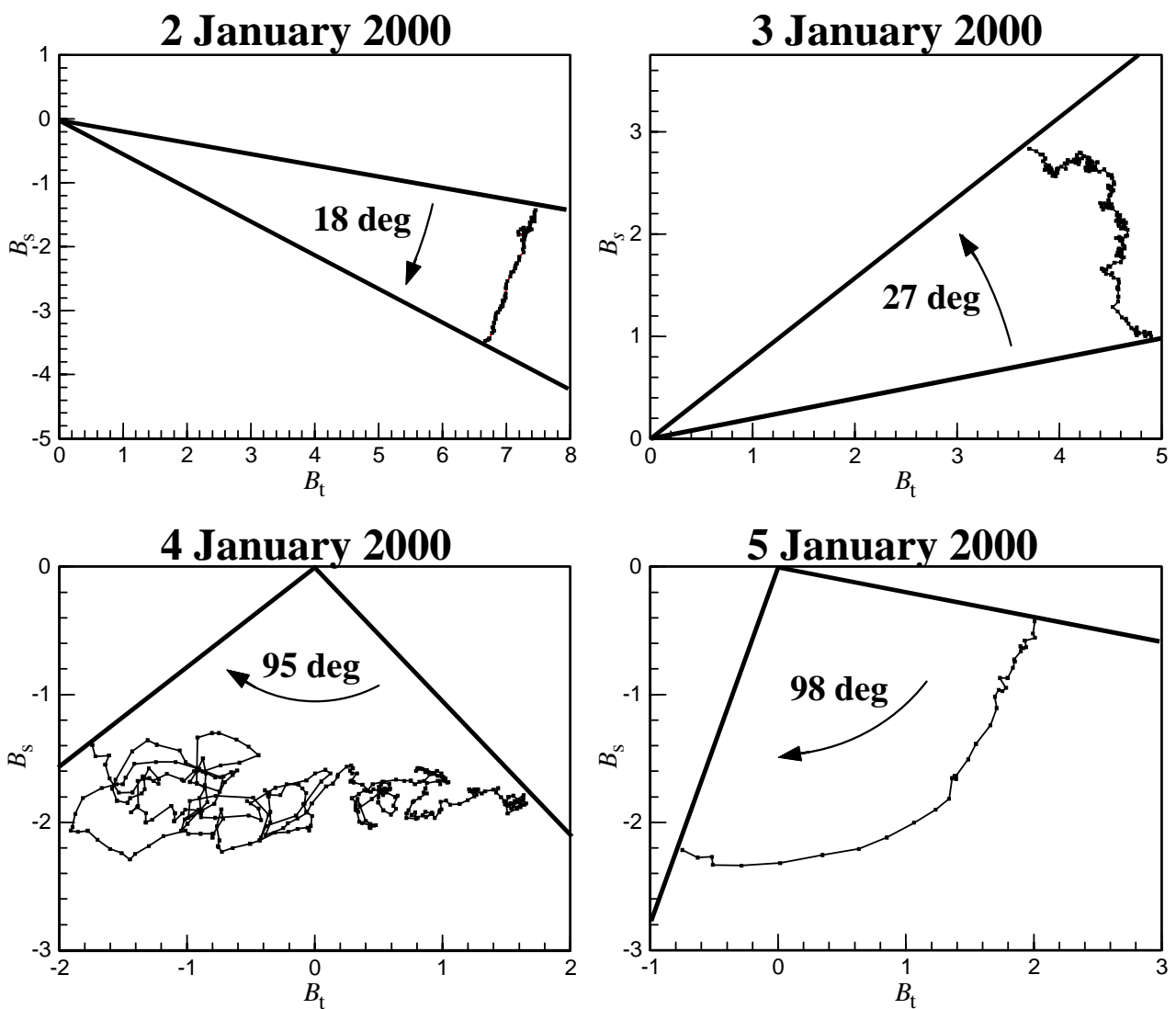

Fig. 14. Through the R-layer of each double discontinuity observed on 2-5 January 2000, the magnetic field rotates by a large angle about the normal direction of the shock surface, while its magnitude remains nearly constant. The rotation angles of the four double discontinuities are $18^{\circ}, 27^{\circ}, 95^{\circ}$, and $98^{\circ}$, respectively.

\section{Appendix}

The normal direction of slow shock layer

A double discontinuity is a compound structure composed of a slow shock layer and an adjoining rotational discontinuity layer on the postshock side. We use the method of Lagrange multipliers to calculate the normal direction of the shock surface along which the variation for the magnetic field data through the slow shock layer is a minimum.

In each study, we have $N$ vector magnetic field data. Within the finite thickness of a typical slow shock layer, we deal with a few hundred of high-resolution data $\boldsymbol{B}$. Let $\langle\boldsymbol{B}\rangle$ be the average, let

$\boldsymbol{b}=B-\langle B\rangle$

be the deviation of the vector magnetic field data, and let

$\boldsymbol{n}=x_{1} \mathbf{i}+x_{2} \mathbf{j}+x_{3} \boldsymbol{k}$

be the unit normal vector. $x_{1}, x_{2}, x_{3}, b_{1}, b_{2}$, and $b_{3}$ are components of $\boldsymbol{n}$ and $\boldsymbol{b}$ in GSE coordinates. The 3 unknown variables, $x_{1}, x_{2}, x_{3}$, must satisfy a constraint function

$f_{1}=x_{1}^{2}+x_{2}^{2}+x_{3}^{2}-1=0$
The square of the total deviation along $n$-direction for the set of the magnetic field data through the interior of the slow shock layer is

$\sigma=\sum_{N}\left(x_{1} b_{1}+x_{2} b_{2}+x_{3} b_{3}\right)^{2}$

Let $\lambda$ be a Lagrange multiplier and let

$f_{k+1}=\frac{\partial \sigma}{\partial x_{k}}+\lambda \frac{\partial f_{1}}{\partial x_{k}} \quad(k=1,3)$

Then solve the system of 4 equations

$f_{k}=0 \quad(k=1,4)$

The solution determines the $n$-direction along which $\sigma$ is a minimum. Currently many routines are available for solving the system of nonlinear equations.

For the double discontinuity observed on 14 February 1994, the Geotail spacecraft crossed the slow shock layer between 17:14:55 and 17:15:41. The number of vector magnetic field data is $N=735$. The solution for analysis of minimal variation gives the three directional angles of the unit normal vector $\cos ^{-1} x_{1}=120^{\circ}, \cos ^{-1} x_{2}=139^{\circ}$, and $\cos ^{-1} x_{3}=115^{\circ}$. 
Acknowledgements. We thank the referees for their helpful comments. We particularly thank S. Kokubun for the Geotail magnetic field data. This work at the Catholic University of America was supported by DOE grant DE-FG02-00ER54582.

Topical Editor G. Chanteur thanks E. J. Smith and another Referee for their help in evaluating this paper.

\section{References}

Abraham-Shrauner, B. and Yun, S. H., Interplanetary shocks seen by Ames Plasma Probe on Pioneer 6 and 7, J. Geophys. Res., 81, 2097, 1976.

Burlaga, L. F. and Chao, J. K., Reverse and forward slow shocks in the solar wind, J. Geophys. Res., 76, 7516, 1971.

Chao, J. K. and Olbert, S., Observation of slow shocks in interplanetary space, J. Geophys. Res., 75, 6394, 1970.

Colburn D. S. and Sonett, C. P., Discontinuity in the solar wind, Space Sci. Rev., 5, 439, 1966.

Feldman, W. C., Schwartz, S. J., Bame, S. J., Baker, D. N., Birn, J., Gosling, J. T., Hones, Jr., E. W., McComas, D. H., Slavin, J. A., Smith, E. J., and Zwickl, R. D., Evidence for slow-mode shocks in the deep geomagnetic tail, Geophys. Res. Lett., 11, 599, 1984.

Feldman, W. C., Baker, D. N., Bame, S. J., Birn, J., Gosling, J. T., Hones, Jr., E. W., and Schwartz, S. J., Slow mode shocks: A semipermanent feature of the distant geomagnetic tail, J. Geophys. Res., 90, 233, 1985.

Feldman, W. C., Tokar, R. L., Birn, J., Hones, Jr., E. W., Bame, S. J., and Russell, C. T., Structure of a slow mode shock observed in plasma sheet boundary layer, J. Geophys. Res., 92, 83, 1987.

Friedman, M. A., Russell, C. T., Gosling, J. T., and Thomsen, M. F., Noncoplanar component of the magnetic field at low Mach number shocks, J. Geophys. Res., 95, 2441, 1990.

Gosling, J. T., Winske, D., and Thomsen, M. F., Noncoplanar magnetic fields at collisionless shocks: a test of a new approach, J. Geophys. Res., 93, 2735, 1988.

Jones, F. C. and Allison, D. C., Noncoplanar magnetic field fields, shock potentials, and ion deflection, J. Geophys. Res., 92, 11, 205, 1987.

Kokubun, S., Yamamoto, T., Acuna, M. H., Hayashi, K., Shiokawa, K., and Kawano, H., The GEOTAIL magnetic field experiment, J. Geomagn. Geoelectr., 46, 7, 1994.

Lee, L. C., Wu, B. H., Chao, J. K., Lin, C. H., and Li, Y., Formation of a compound slow shock-rotational discontinuity structure, J. Geophys. Res., 105, 13, 045, 2000.

Lepping, R. P., Acuna, M. H., Burlaga, L. F., Farrell, W. M., Slavin, J. A., Schatten, K. H., Mariani, F., Ness, N. F., Neubauer, F. M., Whang, Y. C., Byenes, J. B., Kennon, R. S., Panetta, P. V., Scheifele, J., and Worley, E. M., The WIND Magnetic Field Investigation, Space Sci. Rev., 71, 207, 1995.

Mukai, T., Machida, S., Saito, Y., Hirahara, M., Terasawa, T., Kaya,
N., Obara, T., Ejiri, M., and Nishida, A., The low energy particle (LEP) experiment onboard the Geotail satellite, J. Geomagn. Geoelectr., 46, 669, 1994.

Petschek, H. E., Magnetic field annihilation, in AAS-NASA Symposium on the Physics of Solar Flares, (Ed.) Hess, W. N., NASA Sp-50, pp. 425-439, 1964.

Priest, E. R., Solar magnetohydrodynamics, pp. 351-357, D. Reidel Publ. Co., Dordrecht, 1982.

Richter, A. K., Rosenbauer, H., Neubauer, F. M., and Ptitsyna, N. G., Solar wind observations associated with a slow-forward shock wave at 0.31 AU, J. Geophys. Res., 90, 7581, 1985.

Saito, Y., Mukai, T., Terasawa, T., Nishida, A., Machida, S., Hirahara, M., Maezzawa, K., and Yamamoto, T., Slow-mode shocks in the magnetotail, J. Geophys. Res., 100, 23, 567, 1995.

Saito, Y., Mukai, T., Terasawa, T., Nishida, A., Machida, S., Kokubun, S., and Yamamoto, T., Foreshock structure of the slowmode shocks in the Earth's magnetotail, J. Geophys. Res., 101, 13, 267, 1996.

Seon, J., Frank, L. A., Paterson, W. R., Scudder, J. D., Coroniti, F. V., Kokubun, S., and Yamamoto, T., Observations of a slowmode shock at the lobe-plasma sheet boundary in Earth's distance magnetotail, Geophys. Res. Lett., 22, 2981, 1995.

Seon, J., et al., Observations of slow-mode shocks in Earth's distant magnetotail with the Geotail spacecraft, J. Geophys. Res., 12, 27 , 383, 1996.

Smith, E. J., Slavin, J. A., Tsurutani, B. T., Feldman, W. C., and Bame, S. J., Slow mode shocks in the Earth's magnetotail: ISEE 3, Geophys. Res. Lett., 11, 1054, 1984.

Song, P., Russell, C. T., and Thomsen, M. F., Slow mode transition in the frontside magnetosheath, J. Geophys. Res., 97, 8295, 1992.

Sonnerup, B. U. Ö., Magnetic-field re-connexion in a highly conducting incompressible fluid, J. Plasma Phys., 4, 161, 1970.

Thomsen, M. F., Gosling, J. T., Bame, S. J., Livesey, W. A., and Russell, C. T., On the noncoplanarity of the magnetic field within a fast collisionless shock, J. Geophys. Res., 92, 2305, 1987.

Vasyliunas, V. M., Theoretical models of magnetic field line merging, Rev. Geophys. Space Phys., 13, 303, 1975.

Whang, Y. C., Zhou, J., Lepping, R. P., and Ogilvie, K. W., Interplanetary slow shock observed from Wind, Geophys. Res. Lett., 23, 1239, 1996.

Whang, Y. C., Fairfield, D., Smith, E. J., Lepping, R. P., Kokubun, S., and Saito, Y., Observa- tions of double discontinuities in the magnetotail, Geophys. Res. Lett., 24, 3153, 1997.

Whang, Y. C., Zhou, J., Lepping, R. P., Szabo, A., Fairfield, D., Kokubun, S., Ogilvie, K. W., and Fitzenreiter, R., Double discontinuity: A compound structure of slow shock and rotational discontinuity, J. Geophys. Res., 103, 6513, 1998a.

Whang, Y. C., Larson, D., Lepping, R. P., Lin, R. P., and Szabo, A., Plasma and magnetic field structure of a slow shock: Wind observations in interplanetary space, Geophys. Res. Letters, 25, $2625,1998 b$ 